\title{
Paper-based electroanalytical strip for user-friendly blood glutathione detection
}

\author{
Maria Rita Tomei, Stefano Cinti*, Nicolò Interino, Valeria Manovella, Danila Moscone, \\ Fabiana Arduini* \\ Department of Chemical Science and Technologies, University of Rome “Tor Vergata”, Via della Ricerca Scientifica 1, o0133 Rome, Italy
}

\section{A R T I C L E I N F O}

\section{Keywords:}

Screen-printed electrodes

Wax printing

Reagent-free

Self-care

Blood

\begin{abstract}
A B S T R A C T
Paper-based devices are always more gaining a relevant position in the field of sensors. The continuous demand for affordable, simple, sustainable, and portable devices, is making paper as the ideal basis towards the realization of analytical tools for the easy self-testing. In this work, we demonstrate, for the first time, the development of a disposable paper-based printed electroanalytical strip for reliable, rapid, and high-throughput detection of glutathione in blood. The detection is based on the thiol-disulfide exchange reaction, which produces a detectable compound easily oxidizable at a Prussian Blue/carbon black nanocomposite involving a favorable low-interference overpotential. This nanocomposite is mixed within a carbon-based conductive ink and successively screen-printed onto a wax-patterned filter paper. The employment of paper provides a reagent-free device, as a consequence of the reagents pre-loading within the testing area. After the experimental conditions have been optimized, glutathione has been detected up to $10 \mathrm{mM}$, with a detection limit of $60 \mu \mathrm{M}$, and a sensitivity of $(0.102 \pm 0.005) \mu \mathrm{A} / \mathrm{mM}$. This sensor showed satisfactory repeatability (relative standard deviation equal to $10 \%$, for detection of glutathione $1 \mathrm{mM}$ ), especially by considering the hand-made manufacturing process. The "real-world" applicability of this strip has been evaluated by quantifying blood glutathione at physiological levels and by recovery studies achieving satisfactory values.
\end{abstract}

\section{Introduction}

Glutathione, a tripeptide formed by cysteine, glutamic acid, and glycine, is the most abundant antioxidant thiol capable to regulate intracellular redox homeostasis. Its reduced form is among the most exploited antioxidant shields in opposing free radicals and toxins [1,2]. Glutathione level within the mammalian cells is greatly significant: in fact, abnormal levels of blood glutathione can be correlated to several diseases including Alzheimer, Parkinson, diabetes, and various cancers [3-6]. Because its monitoring is of undisputed importance for clinical practice, the development of point-of-care device would be very useful to overcome some limitations often bound to time-consuming procedures such as the requirement of laboratory set-up, skilled personnel, and expensive instrumentations. In clinical chemistry, glutathione has been principally measured by using ultraperformance liquid chromatography (UPLC)-mass spectrometry [7], high performance liquid chromatography (HPLC)-electrospray tandem mass spectrometry $[8,9]$, and gas chromatography-mass spectrometry (GC-MS) [10]. Although these techniques are characterized by satisfactory specificity and sensitivity in biological fluids, they are strongly laboratory-dependent. In addition, chromatographic methods usually require sample derivatization. Alternatively, some "lighter" approaches have been proposed for the detection of glutathione, including electrochemiluminescence [11], fluorescence [12], colorimetry [13], electroanalysis [14], and Raman scattering [15]. However, these methods are characterized by certain limitations: external sources (fluorescence), sensitivity (colorimetry), experimental set-up (electrochemiluminescence, Raman scattering).

Among the above-cited approaches, electroanalysis coupled to screen-printed electrodes offers obvious advantages for their miniaturized size and tiny amount of sample required. In fact, only few $\mu \mathrm{L}$ of blood are required to perform the analysis, such as in well-known glucose strips, when compared with $\mathrm{mL}$ of human blood specimens usually required for clinical practice. An additional advantage is related to the instruments involved, being the cost and the size of instrument well suitable for cost-effective and on-site analysis. Recently, the research activity in the electrochemical miniaturized devices has highlighted the outstanding features of paper in terms of cost-effectiveness,

\footnotetext{
* Corresponding authors.

E-mail addresses: stefano.cinti@uniroma2.it (S. Cinti), fabiana.arduini@uniroma2.it (F. Arduini).
} 
customization, and easily disposability, boosting the use of paper in the development of smart electrochemical sensors [16-21]. For instance, the customization of devices can be easily accomplished by using different types of paper (e.g. office paper [22], carbon tape [23], cotton [24,25], filter paper [26]). In case of office-paper, a drop analysis is carried out; instead, in case of filter or chromatography paper, the solution goes through the cellulose network. For microfluidic systems, filter or chromatography paper is usually employed because the porosity of the paper is exploited for moving the solution without the required of any additional pump. In addition, the porous structure allows for loading all the reagents necessary for the specific assay before the measurement, delivering the so-called "reagent-free" devices. The porosity entails also for blocking gross impurities present in the real matrices, avoiding electrode fouling. In the field of paper-based electrochemical sensors, several analytical tools have been reported in literature for the detection of different analytes including chloride [27], nerve agents [28], heavy metals [29,30], bisphenol A [31], lactate [32], and ascorbic acid [33].

In the case of paper-based sensors, at our knowledge, the only one reported for glutathione detection has been developed by Henry group, and it is based on a colorimetric transduction. In detail, they designed an elegant approach for glutathione detection in serum based on the variation of the silver nanoparticle color, due to their aggregation in presence of glutathione, which turned the solution from orange to deep red [34]. However, the colorimetric detection is able to detect glutathione in serum but not in whole blood, being affected by color or/ and the turbidity of the sample. In addition, glutathione is mainly present in erythrocytes at mM level instead of $\mu \mathrm{M}$ level of serum [35], thus the detection of glutathione in whole blood is an important task. To address this issue, glutathione can be electrochemically detected by following its direct oxidation at electrode surface by applying a high overpotential, e.g. 0.8-1 V [36,37]. However, this direct detection allows for poor specificity because real matrices are plenty of species oxidizable at that working potential. Herein, we designed an electrochemical paper-based sensor for detection of glutathione in blood sample exploiting thiol-disulfide exchange reaction to detect glutathione at low applied potential.

Briefly, the process involves the attack by the thiol glutathione (GSH) to the disulfide cystamine (RSSR), breaking the S-S bond and producing a mixed disulfide (GSSR), with the release of the electrochemically detectable thiol cysteamine (RSH). The cysteamine produced in ratio 1:1 with glutathione, is then detected at a low applied potential thanks to the electrocatalytic properties of Prussian Blue towards the oxidation of cysteamine, accordingly the following reactions [38] (Eqs. (1a)-(1c)):

$\mathrm{GSH}+$ cystamine $(\mathrm{RSSR}) \rightleftharpoons$ cysteamine $(\mathrm{RSH})+(\mathrm{GSSR})$

$\mathrm{PB}+\mathrm{RSH} \rightleftharpoons \mathrm{PW}+\mathrm{RSSR}$

$\mathrm{PW} \rightleftharpoons \mathrm{PB}+\mathrm{e}^{-}$

In detail, after the thiol-disulfide exchange reaction (Eq. (1a)), the cysteamine reacts with PB to give the cystamine (oxidized form of cysteamine) and Prussian White (reduced form of Prussian Blue) (Eq. (1b)). The reduced form of Prussian Blue (Prussian White) is consequently oxidized at the electrode surface, giving an oxidative current which is proportional to the amount of glutathione present in the analyzed sample.

This approach was conceived because Prussian Blue (the electrochemical mediator) is not only capable to measure cysteamine at a working potential much lower than $1 \mathrm{~V}$ with reduction of electroactive interferences, but also it overcomes the fouling problem which is a typical drawback of thiol oxidative-based reaction. In order to simplify both the manufacturing and the utilization of this paper-based device, various strategies have been adopted. Prussian Blue nanoparticles, synthesized onto carbon black, have been mixed together with the conductive ink and successively screen-printed onto waxed filter paper. To make the entire system reagent-free, buffered cystamine has been loaded onto the testing area where the electrodes have been previously printed. Herein, the first electroanalytical paper-based device for blood glutathione detection has been designed and developed. This represents an entirely reagent-free tool for minimally invasive analysis in tiny amount of sample $(5 \mu \mathrm{L})$. The word "reagent-free" is associated with the measurement at the level of non-specialized users: end-users are only asked to drop a tiny amount of sample onto the testing area where all the reagents have been previously loaded during the manufacture. This user-friendly, low-cost, and portable paper-based tool for blood glutathione self-test has been further improved by the optimization of the analytical parameters ( $\mathrm{pH}$, applied potential, cystamine concentration) and of the blood hemolysis strategies.

\section{Experimental}

\subsection{Apparatus and reagents}

Potassium chloride, potassium ferricyanide, ferric chloride, boric acid, glacial acetic acid, orto-phosphoric acid $85 \%$, potassium dihydrogen phosphate, glutathione, cystamine, and cysteamine were purchased from Sigma Aldrich. All the solutions were prepared in distilled water. Blood samples were provided by one of the authors, Valeria Manovella. Chronoamperometry measurements were carried out using a portable EmStat ${ }^{3}$ Instrument (PalmSens, Netherlands) connected to a laptop. Blood hemolysis was conducted with ultrasonic bath (LBS2, Falc).

\subsection{Fabrication of paper-based strip}

To fabricate the paper-based sensor for glutathione detection, four steps (i, ii, iii, iv) were followed:

i) Testing area was designed with a drawing software (Adobe Illustrator) and printed onto filter paper $\left(67 \mathrm{~g} / \mathrm{m}^{2}\right.$, Cordenons, Italy) by using an office wax printer (ColorQube 8580 , Xerox, USA).

ii) Waxed paper was cured in an oven at $100^{\circ} \mathrm{C}$ for $2 \mathrm{~min}$, to create specific hydrophobic areas.

iii) Electrodes were manually screen-printed by using a polymeric squeegee and masks. $\mathrm{Ag} / \mathrm{AgCl}$ ink (Electrodag $6038 \mathrm{SS}$, Acheson, Italy) was used for the reference electrode, and graphite ink (Electrodag 421, Acheson, Italy) modified with 5\% (w/w) Prussian Blue/carbon black powder for the working and counter electrode. $20 \mathrm{~min}$ in the oven at $60{ }^{\circ} \mathrm{C}$ were enough to cure the inks.

iv) $5 \mu \mathrm{L}$ of the working solution containing cystamine dissolved in Britton Robinson buffer were pipetted onto the hydrophilic testing area.

The entire design of the paper-based screen-printed electrode is reported in the supplementary information file (Fig. S1) and the presence of waxed area allows to confine the solution in the delimitated electrochemical cell area, avoiding its diffusion towards electric contacts.

\subsection{Glutathione detection}

The glutathione detection was carried out in amperometric mode by simply dropping $5 \mu \mathrm{L}$ of sample on sensor testing area, which is the volume to wet the electrochemical cell without any leakage of the sample. The quantification of glutathione is carried out in amperometric mode, by measuring cysteamine at low applied potential $(0.3 \mathrm{~V}$ vs $\mathrm{Ag} / \mathrm{AgCl}$ ), because cysteamine is the by-product of thiol-disulfide exchange reaction between glutathione and cystamine (Eqs. (1a)-(1c)).

As reported in Fig. 1, all the reagents needed for the detection of glutathione (i.e. cystamine $0.05 \mathrm{M}$ in Britton Robinson buffer solution 


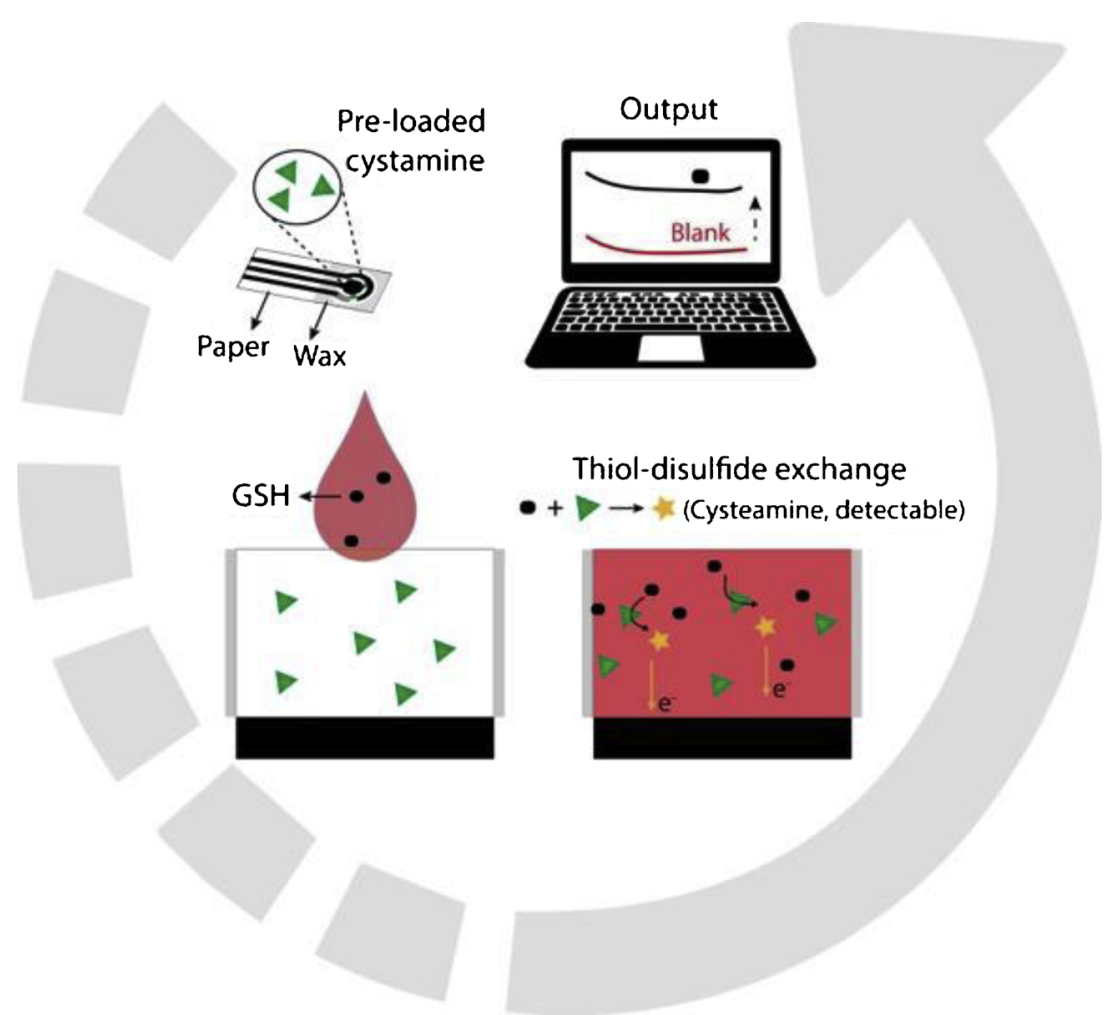

Fig. 1. Schematic representation of the detection mechanism of the paper-based device.

$\mathrm{pH}=9$ ) were pre-loaded in the testing area in order to realize a reagent free device. After the drying step, the sample was added on the back of testing area to overcome matrix effect during the glutathione detection in blood, exploiting the filtering properties of paper. After the thioldisulfide exchange reaction, the resulting current (depending on the glutathione level) was recorded by using a portable potentiostat interfaced to a laptop. In detail, the current values were sampled at $100 \mathrm{~s}$ to make the fitting curves. For glutathione detection in real samples, the blood collection has been carried out by a medical doctor from a colleague, following Ethical guidelines provided by Occupational health section, University of Oxford [39]. We supplied Donor Information Sheet and Donor Consent form to the donor.

\section{Results and discussions}

\subsection{Principle of the glutathione detection by thiol-disulfide exchange reaction and its optimization}

The electrochemical detection of thiols is often limited by the high overpotential needed to make the reaction happen. Typically, an applied potential close to $1 \mathrm{~V}$ is sufficient to oxidize a lot of compounds at the electrode surface, decreasing the specificity of the method. To overcome this problem, we chose another strategy: glutathione participates to the thiol-disulfide reaction in presence of cystamine. Successively, the byproduced cysteamine can be easily and selectively detected at the electrode at low applied potential, taking into account the molecules present in whole blood. In this work, we decided to take advantage of a nanocomposite produced by the combination of Prussian Blue and carbon black. As already reported in our previous paper, carbon black acts as a nanosized support for the synthesis of Prussian Blue nanoparticles starting from its precursors, i.e. potassium ferricyanide and ferric chloride [40]. Prussian Blue is widely known for its role of artificial peroxidase towards the reduction of hydrogen peroxide at low interfering potential [41,42]. Briefly, the by-produced cysteamine (following the thiol-disulfide reaction between glutathione and cystamine) is oxidized while Prussian Blue (PB) is converted in its reduced form, Prussian White (PW). PW is then oxidized back to PB at the screen-printed electrode (SPE), making it able to electrocatalyze the oxidation of additional cysteamine molecules (Eqs. (1a)-(1c)). Experimentally, as a consequence of cysteamine oxidation, for cyclic voltammetry studies, the PB/PW redox couple reveals an improvement of the current peak related to the oxidation from $\mathrm{PW}$ to $\mathrm{PB}$, coupled to a decrease of the peak related to the opposite reaction, which converts $\mathrm{PB}$ to $\mathrm{PW}$, as shown in Fig. 2A.

To develop a sensitive device, the experimental parameters need to be optimized, starting from the working conditions of cysteamine detection, which is the thiol-disulfide exchange reaction product. The first parameter optimized was the potential applied during the chronoamperometry measurements. The applied potential should be the lowest capable to provide the most effective oxidation of cysteamine. A common issue related to the electrochemical methods is represented by the possibility to oxidize/reduce other species present in the sample. The use of PB or other electrochemical mediators such as cobalt phathalocyanine, decreases this chance, especially when compared with those methods involving carbon-based electrodes [28,43]. As shown in Fig. 2B, the effect of the applied potential was investigated in the anodic range comprised between 0.1 and $0.4 \mathrm{~V}$. Although the mean currents recorded by the application of $0.4 \mathrm{~V}$ appears slightly higher in respect to the others, the application of $0.3 \mathrm{~V}$ is consistent with the best compromise in terms of sensitivity and repeatability. After the optimization of the applied potential, the effect of $\mathrm{pH}$ was also evaluated, being the $\mathrm{pH}$ affects the oxidation of thiols. As expected, at higher $\mathrm{pH}$ a more sensitive response has been observed because the sulfur moiety of the cysteamine species becomes deprotonated, rendering the cysteamine more easily oxidizable, as in the case of homocysteine [37]. For further studies, a pH of 9 was chosen as the optimal one, considering both the current intensity and the repeatability (Fig. 2C). It should be noted that, even if alkaline environment is required to obtain the optimal analytical performance, the end-user is not going to consider this experimental parameter during measurements, i.e. adjusting $\mathrm{pH}$. Indeed, the use of porous paper impregnated with all the required 

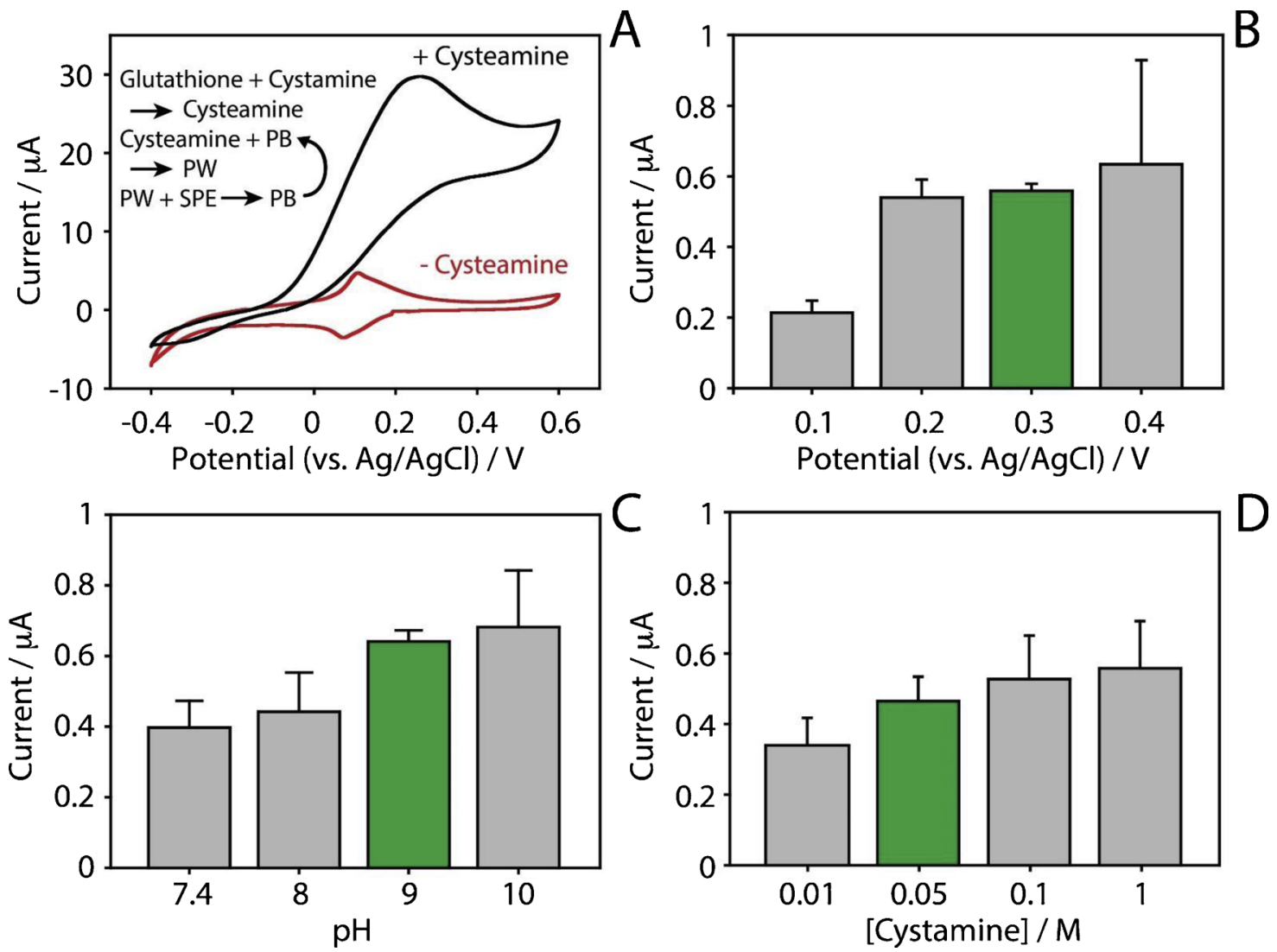

Fig. 2. A) Cyclic voltammetry performed in absence (red line) and in presence (black line) of $10 \mathrm{mM}$ cysteamine prepared in $50 \mathrm{mM}$ phosphate buffer (pH 7.4 ) containing $100 \mathrm{mM}$ of $\mathrm{KCl}$; B) Optimization of the working potential, $0.1,0.2,0.3$ and $0.4 \mathrm{~V}$. Experimental conditions: phosphate buffer at $\mathrm{pH}=7.4$, [cysteamine] $=10 \mathrm{mM}$; C) Optimization of the $\mathrm{pH}$, from 7.4 to 10 in Britton Robinson buffer. Experimental conditions: $\mathrm{E}=0.3 \mathrm{~V}$, [cysteamine] $=10 \mathrm{mM}$; D) Optimization of cystamine concentration from 0.01 to $1 \mathrm{M}$ using $5 \mathrm{mM}$ glutathione. Experimental conditions: $\mathrm{E}=0.3 \mathrm{~V}$; $\mathrm{pH}=9$. All the measurements were performed by pipetting a 5- $\mu \mathrm{L}$ drop of distilled water containing the analyte on the back of the paper-based strip. Each bar is the result of three different strips tested in chronoamperometry mode (For interpretation of the references to colour in this figure legend, the reader is referred to the web version of this article.).

reagents for a certain assay, furnishes a great advantage which relays on the addition of only few $\mu \mathrm{L}$ of the sample, delivering "reagent free" analytical tool. After the $\mathrm{pH}$, last parameter optimized was the cystamine concentration adsorbed on the testing area of the paper-based strip. In presence of a fixed concentration of glutathione, i.e. $5 \mathrm{mM}$, the level of cystamine was evaluated in the 0.01-1 M range. As reported in Fig. 2D, a concentration of $0.05 \mathrm{M}$ cystamine was effective with the lowest concentration yielding the best compromise in terms of sensitivity and repeatability, thus this value has been selected for the further studies. As shown, an ulterior increase of cystamine did not produce any significant improvement of the signal.

\subsection{Glutathione detection in standard solutions}

After optimization of applied potential, $\mathrm{pH}$, and cystamine concentration, the paper-based strip was challenged in presence of increasing concentrations of glutathione. To perform the chronoamperometric measurements, the testing area was wetted with $5 \mu \mathrm{L}$ of glutathione solutions prepared in distilled water. Glutathione was detected up to $10 \mathrm{mM}$ as reported in Fig. 3 .

The recorded signals increased linearly with the increase of glutathione concentration (0-0.25-0.5-1-2-5-10 mM). With the optimized parameters, the correlation between the anodic current and the concentration of glutathione was described by the following equation: $\mathrm{y}=$ $(0.04 \pm 0.02)+(0.102 \pm 0.005) x\left(R^{2}=0.956\right)$ where $y$ indicates the current in $\mu \mathrm{A}$ and $\mathrm{x}$ states the concentration of glutathione in $\mathrm{mM}$. The linear range $\left(R^{2}=0.956\right)$ is observed in the range up to $10 \mathrm{mM}$, and the detection limit (LOD), calculated as the signal-to-noise ratio

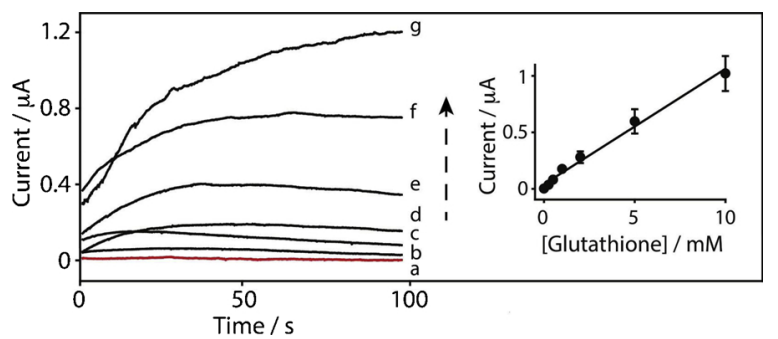

Fig. 3. Chronoamperometry detection recorded in absence (a, red line) and in presence of (b) 0.25 , (c) 0.5 , (d) 1 , (e) 2.5 , (f) 5 , and (g) $10 \mathrm{mM}$ glutathione. Right inset: Calibration curve $(n=3)$. All the tested SPEs have been pre-loaded during the production process with $5 \mu \mathrm{L}$ of $0.05 \mathrm{M}$ cystamine dissolved in $50 \mathrm{mM}$ Britton Robinson buffer ( $\mathrm{pH}$ 9). The applied potential was $0.3 \mathrm{~V}$ and the currents values were collected in the chronoamperograms at $100 \mathrm{~s}$ (For interpretation of the references to colour in this figure legend, the reader is referred to the web version of this article.).

equal to $(\mathrm{S} / \mathrm{N}=3)$, resulted equal to $60 \mu \mathrm{M}$. The relative standard deviation (RSD) calculated for three measurements of $1 \mathrm{mM}$ glutathione was found equal to $10 \%$. This is a strict consequence of the robustness coupled to the strips manufacturing process.

\subsection{Storage stability}

In order to evaluate the applicability of the developed sensor, a stability study was carried out testing the sensor towards glutathione within 1 month. In detail, sensors were pre-loaded with all the reagents needed for glutathione detection and stored at room condition. The pre- 


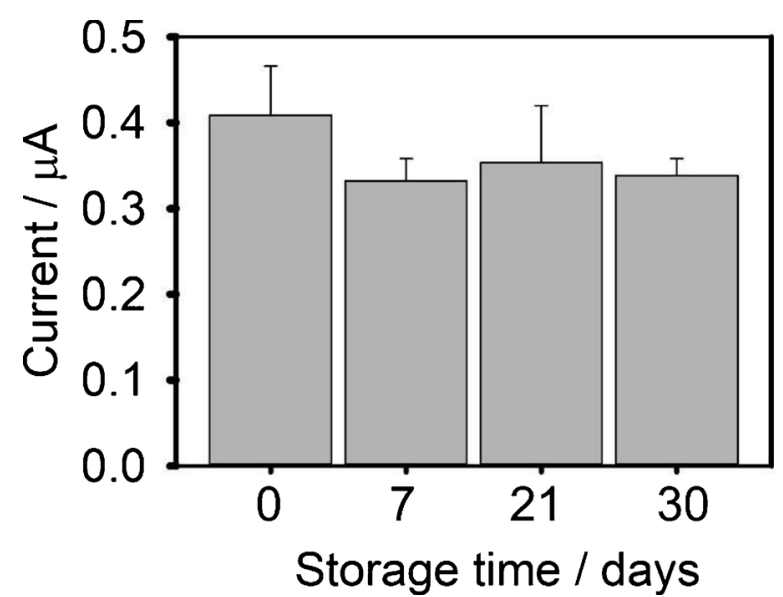

Fig. 4. Storage stability study maintaining at room temperature the paperbased sensors loaded with all reagents needed for the measurement.

loaded sensors were tested using a solution of glutathione $3 \mathrm{mM}$ at zero time and after 7,21 , and 30 days. The values obtained are reported in Fig. 4, which are the results of triplicate measurements for each time, demonstrating the valuable storage stability of the sensor at room temperature for at least 1 month.

\subsection{Evaluation of the interference}

Prior to work with matrix i.e. blood, the detection mechanism should be evaluated in presence of common species that can be founded

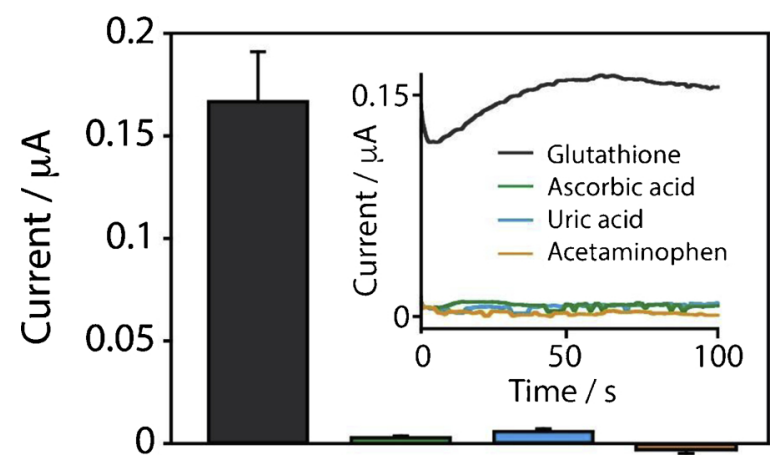

Fig. 5. Histograms related to the response of the paper-based strip in presence of (grey line) $1 \mathrm{mM}$ glutathione, (green line) $50 \mathrm{mM}$ ascorbic acid, (sky blue) $50 \mathrm{mM}$ uric acid, and (orange) $50 \mathrm{mM}$ acetaminophen. The histograms are the result of three different strips, and the experimental conditions equal to those reported in caption of Fig. 3. Inset: chronoamperometric curves (For interpretation of the references to colour in this figure legend, the reader is referred to the web version of this article.). in this complex media. For instance, in blood, the presence of easily oxidizable compounds such as ascorbic acid, uric acid, and acetaminophen, could be consistent with false positive signals, because these species can be oxidized at the electrode surface in addition to the glutathione. The sensor strip was tested in presence of these interfering species as shown in Fig. 5.

As shown in Fig. 5, the presence of these interfering species does not significantly affect glutathione detection. The concentrations considered for this study have been selected by taking into account their physiological levels in blood at $\mu \mathrm{M}$ level [44-46]. Beside these interfering species, it should be noted that the thiol-disulfide reaction could be sensitive to the presence of other thiols, i.e. cysteine: the involved reaction is not specific for glutathione, but it interests all the thiols. However, as widely reported in literature, the erythrocyte concentration of other thiols is less of around 1\% in respect to the glutathione amount $[14,35,47,48]$, avoiding the overestimation of glutathione content in erythrocyte.

It means, accordingly with the analytical performance of the present method (LOD of $60 \mu \mathrm{M}$ ), that the non-glutathione thiols are not capable to affect the signal that arises from glutathione in the $\mathrm{mM}$ range.

\subsection{Detection of blood glutathione}

The ultimate paper-based device was then applied towards the detection of glutathione in blood samples. The challenge in developing a portable test for blood glutathione detection is because this metabolite is almost entirely stored in the erythrocytes. Prior to begin with the real matrix measurements, we evaluated two different easy strategies for treating blood: i) the dilution with distilled water (ratio 1:2 v/v) and ii) a sonication ( $59 \mathrm{kHz}$ for $10 \mathrm{~min}$ ) followed by the dilution with distilled water (ratio $1: 2 \mathrm{v} / \mathrm{v}$ ). These treatments have been selected being the dilution and/or sonication usually applied for lysis of erythrocytes [49-51]. Using these treatments we found values of current equal to $110 \mathrm{nA}(\mathrm{RSD} \%=30)$ and $145 \mathrm{nA}(\mathrm{RSD} \%=15)$, respectively. These results demonstrated that the sonication step improves the repeatability and the release of glutathione by erythrocytes, thus, the treatment that comprises dilution and sonication has been selected for the further studies.

Subsequently, blood was spiked with different amounts of glutathione giving the final concentration in the $1-3 \mathrm{mM}$ range. As reported in Fig. 6, the ultimate paper-based device was used to perform the measurements.

Three levels of glutathione added in blood were analyzed and a good correlation was obtained, demonstrating the suitability of the paper-based strip to detect glutathione in a complex matrix like the blood is. The correlation is described by the following equation, $\mathrm{y}=$ $(0.19 \pm 0.02)+(0.119 \pm 0.009) x,\left(R^{2}=0.947\right)$, and a good sensitivity of $0.119 \mu \mathrm{A} / \mathrm{mM}$ is obtained. The repeatability associated to the blood glutathione measurement has been calculated with a RSD equal to $10 \%$ for glutathione $1 \mathrm{mM}$. We would like to stress an important advantage related to the use of paper as the manufacturing substrate for

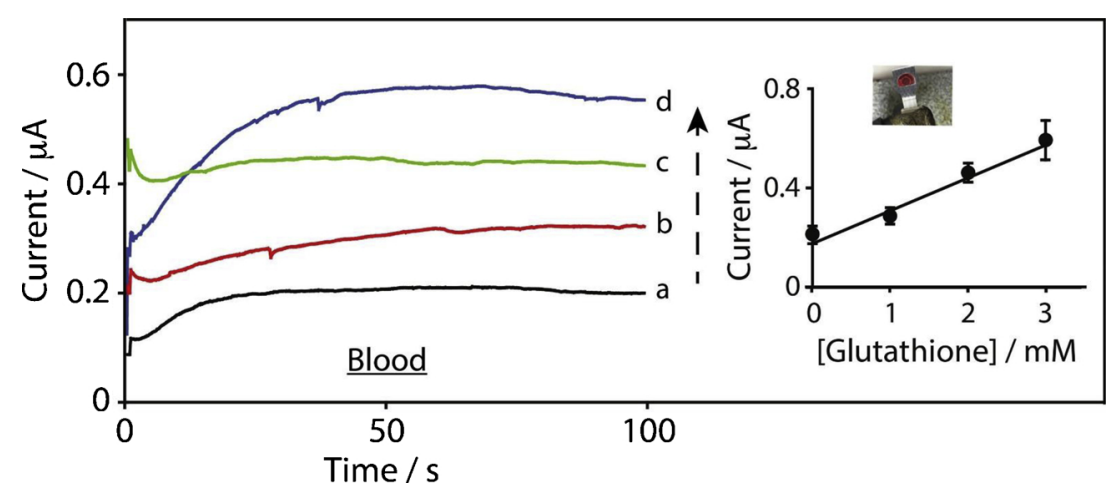

Fig. 6. Chronoamperometry detection recorded in blood after selected treatment in absence (a, black line) and in presence of (b, red line) 1 , (c, green line) 2 , and (d, blue line) $3 \mathrm{mM}$ glutathione. Right inset: Calibration curve $(n=3)$. The experimental conditions are equal to those reported in caption of Fig. 3 (For interpretation of the references to colour in this figure legend, the reader is referred to the web version of this article.). 
printed electrochemical devices: as already demonstrated in our previous papers, the porosity of filter paper is very effective in blocking the gross impurities present in these complex matrices, thus reducing the electrode surface fouling. The main issue of the plastic-based SPEs employed in drop or batch experimental setups, is related to the protein/fat stacking. Filter paper offers an integrated barrier for sample treatment that can be enforced by improving the layers and/or employing a different grade of porosity. Moreover, the accuracy of the method was evaluated accordingly the indications reported in 2002/ $657 / \mathrm{EC}$ concerning the performance of analytical methods and the interpretation of results [52], by spiking blood with known concentration of analyte i.e. glutathione equal to 2 and $3 \mathrm{mM}$. The obtained recoveries were equal to $90 \pm 15 \%$ and $100 \pm 16 \%$, respectively for 2 and $3 \mathrm{mM}$ glutathione $(\mathrm{n}=3)$. The results achieved in terms of trueness and reproducibility (RDS $\%=10 \%$ both in buffer and blood, calculated using glutathione level at $1 \mathrm{mM}$ ), demonstrated the suitability of the developed paper-based sensor for glutathione detection in blood. When compared with the electrochemical sensors developed in literature (Table 1), the proposed sensor is characterized by higher detection limit (i.e. $60 \mu \mathrm{M}$ ), however this sensor is the only one capable to detect glutathione at physiological level without any further addition of reagents during the measurement, but only adding a drop of diluted and sonicated sample onto the paper-strip.

\section{Conclusions}

Herein, we reported the first example of a paper-based electrochemical strip for glutathione detection. The optimized sensing platform is characterized by a detection limit of $60 \mu \mathrm{M}$ and a linearity up to $10 \mathrm{mM}$, using chronoamperometry as technique and $+0.3 \mathrm{~V} \mathrm{vs} \mathrm{Ag} / \mathrm{AgCl}$ as applied potential. The low applied potential and the overcoming of fouling problem for cysteamine detection (the by-product of the reaction between glutathione and cystamine) is due to use of ink-nanomodifier constituted of nanoparticles of Prussian Blue grown onto carbon black, demonstrating the suitability of this paper-based sensor for thiol detection (i.e. cysteamine/glutathione). The accuracy of the platform for glutathione detection was demonstrated by analyzing spiked-blood samples. The results achieved demonstrated the effectiveness of this paper-based sensor for affordable, easy-to-operate, and de-centralized detection of glutathione in blood, with the advantage to be a fully reagent-free analytical tool. This work represents another evidence of the increasing role of paper in the field of electroanalytical devices and its implementation: step-by-step, coupled to easy customization techniques (screen-printing, wax printing, nanomaterial synthesis) the position of paper in the field of sensor is knowledgeably, especially for developing countries where cost-effective and easy to use analytical devices are highly required.

\section{Acknowledgements}

Stefano Cinti acknowledges Fondazione Umberto Veronesi for Postdoctoral Fellowship 2018. Authors thank Julian Ramirez for proofreading the English of the manuscript.

\section{Appendix A. Supplementary data}

Supplementary material related to this article can be found, in the online version, at doi:https://doi.org/10.1016/j.snb.2019.02.082.

\section{References}

[1] Y. Yin, J. Kwon, D. Kim, D. Lee, G. Kim, Y. Hu, J. Ryu, J. Yoon, Cyanine-based fluorescent probe for highly selective detection of glutathione in cell cultures and live mouse tissues, J. Am. Chem. Soc. 136 (2014) 5351-5358.

[2] L.Y. Niu, Y.S. Guan, Y.Z. Chen, L.Z. Wu, C.H. Tung, Q.Z. Yang, BODIPY-based ratiometric fluorescent sensor for highly selective detection of glutathione over cysteine and homocysteine Q.Z, J. Am. Chem. Soc. 134 (2012) 18928-18931. 
[3] C. Lentner, Geigy Scientific Tables, eighth edition, 3 CIBA-Geigy, Basle, Switzerland, 1984.

[4] D.M. Townsend, K.D. Tew, H. Tapiero, The importance of glutathione in human disease, Biomed. Pharmacother. 57 (2003) 145-155.

[5] Y.H. Han, S.H. Kim, S.Z. Kim, W.H. Park, Caspase inhibitor decreases apoptosis in pyrogallol-treated lung cancer Calu-6 cells via the prevention of GSH depletion, Int. J. Oncol. 33 (2008) 1099-1105.

[6] J.C. Harfield, C. Batchelor-McAuley, R.G. Compton, Electrochemical determination of glutathione: a review, Analyst 137 (2012) 2285-2296.

[7] L. Cao, D. Waldon, Y. Teffera, J. Roberts, M. Wells, M. Langley, Z. Zhao, Ratios of biliary glutathione disulfide (GSSG) to glutathione (GSH): a potential index to screen drug-induced hepatic oxidative stress in rats and mice, Anal. Bioanal. Chem. 405 (2013) 2635-2642.

[8] E. Camera, M. Rinaldi, S. Briganti, M. Picardo, S. Fanali, Simultaneous determination of reduced and oxidized glutathione in peripheral blood mononuclear cells by liquid chromatography-electrospray mass spectrometry, J. Chromatogr. B 757 (2001) 69-78.

[9] Z. Jiang, Q. Liang, G. Luo, P. Hu, P. Li, Y. Wang, HPLC-electrospray tandem mass spectrometry for simultaneous quantitation of eight plasma aminothiols: application to studies of diabetic nephropathy, Talanta 77 (2009) 1279-1284.

[10] A. Küster, I. Tea, S. Sweeten, J.C. Rozé, R.J. Robins, D. Darmaun, Simultaneous determination of glutathione and cysteine concentrations and $2 \mathrm{H}$ enrichments in microvolumes of neonatal blood using gas chromatography-mass spectrometry, Anal. Bioanal. Chem. 390 (2008) 1403-1412.

[11] W. Gao, Z. Liu, L. Qi, J. Lai, S.A. Kitte, G. Xu, Ultrasensitive glutathione detection based on lucigenin cathodic electrochemiluminescence in the presence of $\mathrm{MnO}_{2}$ nanosheets, Anal. Chem. 88 (2016) 7654-7659.

[12] Q.Y. Cai, J. Li, J. Ge, L. Zhang, Y.L. Hu, Z.H. Li, L.B. Qu, A rapid fluorescence "switch-on" assay for glutathione detection by using carbon dots-MnO2 nanocomposites, Biosens. Bioelectron. 72 (2015) 31-36.

[13] H. Xu, Y. Wang, X. Huang, Y. Li, H. Zhang, X. Zhong, Hg $2^{+}$-mediated aggregation of gold nanoparticles for colorimetric screening of biothiols, Analyst 137 (2012) 924-931.

[14] F. Ricci, F. Arduini, C.S. Tuta, U. Sozzo, D. Moscone, A. Amine, G. Palleschi, Glutathione amperometric detection based on a thiol-disulfide exchange reaction, Anal. Chim. Acta 558 (2006) 164-170.

[15] A. Saha, N.R. Jana, Detection of cellular glutathione and oxidized glutathione using magnetic-plasmonic nanocomposite-based "Turn-Off" surface enhanced raman scattering, Anal. Chem. 85 (2013) 9221-9228.

[16] A.W. Martinez, S.T. Phillips, G.M. Whitesides, Devices (uPADs)-are a new platform designed for ASSURED, Anal. Chem. 82 (2010) 3-10.

[17] C. Parolo, A. Merkoçi, Paper-based nanobiosensors for diagnostics, Am. Chem. Soc. Rev. 42 (2013) 450-457.

[18] Y. Yang, E. Noviana, M.P. Nguyen, B.J. Geiss, D.S. Dandy, C.S. Henry, Paper-based microfluidic devices: emerging themes and applications, Anal. Chem. 89 (2016) 71-91.

[19] F. Arduini, S. Cinti, V. Scognamiglio, D. Moscone, Paper-based electrochemical devices in biomedical field: recent advances and perspectives, Compr. Anal. Chem. 77 (2017) 385-413.

[20] J. Mettakoonpitak, K. Boehle, S. Nantaphol, P. Teengam, J.A. Adkins, M. Srisa-Art, C.S. Henry, Electrochemistry on paper-based analytical devices: a review, Electroanalysis 28 (2016) 1420-1436.

[21] W.R. de Araujo, C.M. Frasson, W.A. Ameku, J.R. Silva, L. Angnes, T.R. Paixão, Single-step reagentless laser scribing fabrication of electrochemical paper-based analytical devices, Angew. Chem. 129 (2017) 15309-15313.

[22] W.R. de Araujo, T.R.L.C. Paixao, Fabrication of disposable electrochemical devices using silver ink and office paper, Analyst 139 (2014) 2742-2747.

[23] F.J. Gomez, P.A. Reed, D.G. Casamachin, J.R. de la Rosa, G. Chumanov, M.F. Silva, C.D. Garcia, Carbon tape as a convenient electrode material for electrochemical paper-based microfluidic devices (ePADs), Anal. Methods 10 (2018) 4020-4027.

[24] C. Sun, X. Li, Z. Cai, F. Ge, Carbonized cotton fabric in-situ electrodeposition polypyrrole as high-performance flexible electrode for wearable supercapacitor, Electrochim. Acta 296 (2019) 617-626.

[25] S.C. Lin, M.Y. Hsu, C.M. Kuan, H.K. Wang, C.L. Chang, F.G. Tseng, C.M. Cheng, Cotton-based diagnostic devices, Sci. Rep. 4 (2014) 6976.

[26] S. Cinti, D. Talarico, G. Palleschi, D. Moscone, F. Arduini, Novel reagentless paperbased screen-printed electrochemical sensor to detect phosphate, Anal. Chim. Acta 919 (2016) 78-84.

[27] S. Cinti, L. Fiore, R. Massoud, C. Cortese, D. Moscone, G. Palleschi, F. Arduini, Lowcost and reagent-free paper-based device to detect chloride ions in serum and sweat, Talanta 179 (2018) 186-192.

[28] S. Cinti, C. Minotti, D. Moscone, G. Palleschi, F. Arduini, Fully integrated ready-touse paper-based electrochemical biosensor to detect nerve agents, Biosens. Bioelectron. 93 (2017) 46-51.

[29] S. Cinti, B. De Lellis, D. Moscone, F. Arduini, Sustainable monitoring of Zn (II) in biological fluids using office paper, Sens. Actuators B 253 (2017) 1199-1206.

[30] L. Feng, X. Li, H. Li, W. Yang, L. Chen, Y. Guan, Enhancement of sensitivity of paperbased sensor array for the identification of heavy-metal ions, Anal. Chim. Acta 780 (2013) 74-80.

[31] H. Li, W. Wang, Q. Lv, G. Xi, H. Bai, Q. Zhang, Disposable paper-based electrochemical sensor based on stacked gold nanoparticles supported carbon nanotubes for the determination of bisphenol A, Electrochem. Commun. 68 (2016) 104-107.

[32] D.D. Liana, B. Raguse, J.J. Gooding, E. Chow, Recent advances in paper-based sensors, Sensors 12 (2012) 11505-11526.

[33] S. Cinti, N. Colozza, I. Cacciotti, D. Moscone, M. Polomoshnov, E. Sowade, F. Arduini, Electroanalysis moves towards paper-based printed electronics: carbon black nanomodified inkjet-printed sensor for ascorbic acid detection as a case study, Sens. Actuators B 265 (2018) 155-160.

[34] D.M. Cate, W. Dungchai, J.C. Cunningham, J. Volckens, C.S. Henry, Simple, distance-based measurement for paper analytical devices, Lab Chip 13 (2013) 2397-2404.

[35] A. Pastore, G. Federici, E. Bertini, F. Piemonte, Analysis of glutathione: implication in redox and detoxification, Clin. Chim. Acta 333 (2003) 19-39.

[36] R.R. Moore, C.E. Banks, R.G. Compton, Electrocatalytic detection of thiols using an edge plane pyrolytic graphite electrode, Analyst 129 (2004) 755-758.

[37] O. Nekrassova, N.S. Lawrence, R.G. Compton, Analytical determination of homocysteine: a review, Talanta 60 (2003) 1085-1095.

[38] F. Ricci, F. Arduini, A. Amine, D. Moscone, G. Palleschi, Characterisation of Prussian blue modified screen-printed electrodes for thiol detection, J. Electroanal. Chem. 563 (2004) 229-237.

[39] http://www.admin.ox.ac.uk/uohs/policies-guidance/blood/. (Accessed on 26 January 2019).

[40] S. Cinti, F. Arduini, G. Vellucci, I. Cacciotti, F. Nanni, D. Moscone, Carbon black assisted tailoring of Prussian Blue nanoparticles to tune sensitivity and detection limit towards $\mathrm{H}_{2} \mathrm{O}_{2}$ by using screen-printed electrode, Electrochem. Commun. 47 (2014) 63-66.

[41] A.A. Karyakin, E.E. Karyakina, L. Gorton, Amperometric biosensor for glutamate using Prussian blue-based "artificial peroxidase" as a transducer for hydrogen peroxide, Anal. Chem. 72 (2000) 1720-1723.

[42] F. Ricci, G. Palleschi, Sensor and biosensor preparation, optimisation and applications of Prussian Blue modified electrodes, Biosens. Bioelectron. 21 (2005) $389-407$.

[43] S. Cinti, D. Neagu, M. Carbone, I. Cacciotti, D. Moscone, F. Arduini, Novel carbon black-cobalt phthalocyanine nanocomposite as sensing platform to detect organophosphorus pollutants at screen-printed electrode, Electrochim. Acta 188 (2016) 574-581.

[44] S. Park, The effects of high concentrations of vitamin C on cancer cells, Nutrients 5 (2013) 3496-3505.

[45] V. Moatti-Sirat, V. Poitout, M.N. Thome, Y. Gangnerau, Y. Zhang, G. Hu, Reach, reduction of acetaminophen interference in glucose sensors by a composite Nafion membrane: demonstration in rats and man, Diabetologia 37 (1994) 610-616.

[46] R. Mundaca-Uribe, F. Bustos-Ramírez, C. Zaror-Zaror, M. Aranda-Bustos, J. NeiraHinojosa, C. Peña-Farfal, Development of a bienzymatic amperometric biosensor to determine uric acid in human serum, based on mesoporous silica (MCM-41) for enzyme immobilization, Sens. Actuators B 195 (2014) 58-62.

[47] A.F. Banne, A. Amiri, R.W. Pero, Reduced level of serum thiols in patients with a diagnosis of active disease, J. Anti Aging Med. 6 (2003) 327-334.

[48] J. Qian, J. Fang, Q. Zhu, S. Ma, W. Wang, Y. Zheng, F. Ding, Serum protein thiol levels in patients with hospital-acquired acute kidney injury, Kidney Blood Press. Res. 40 (2015) 623-629.

[49] G. Lippi, G. Cervellin, E.J. Favaloro, M. Plebani, In Vitro and in Vivo Hemolysis: An Unresolved Dispute in Laboratory Medicine, Walter de Gruyter, Germany, 2012.

[50] M.A. Boyd, S.M. Tennant, J.H. Melendez, D. Toema, J.E. Galen, C.D. Geddes, M.M. Levine, Adaptation of red blood cell lysis represents a fundamental breakthrough that improves the sensitivity of Salmonella detection in blood, J. Appl. Microbiol. 118 (2015) 1199-1209.

[51] N. Kawai, M. Iino, Molecular damage to membrane proteins induced by ultrasound, Ultrasound in Med, \& Biol. 29 (2003) 609-614.

[52] COMMISSION DECISION of 12 August 2002 implementing Council Directive 96/ 23/EC concerning the performance of analytical methods and the interpretation of results $(2002 / 657 / \mathrm{EC})$.

[53] J. Narang, N. Chauhan, P. Jain, C.S. Pundir, Silver nanoparticles/multiwalled carbon nanotube/polyaniline film for amperometric glutathione biosensor, Int. J. Biol. Macromol. 50 (2012) 672-678.

[54] P. Calvo-Marzal, K.Y. Chumbimuni-Torres, N.F. Höehr, L.T. Kubota, Determination of glutathione in hemolysed erythrocyte with amperometric sensor based on TTFTCNQ, Clin. Chim. Acta 371 (2006) 152-158.

[55] L. Rover Jr., L.T. Kubota, N.F. Höehr, Development of an amperometric biosensor based on glutathione peroxidase immobilized in a carbodiimide matrix for the analysis of reduced glutathione from serum, Clin. Chim. Acta 308 (2001) 55-67.

[56] A. Salimi, S. Pourbeyram, Renewable sol-gel carbon ceramic electrodes modified with a Ru-complex for the amperometric detection of l-cysteine and glutathione, Talanta 60 (2003) 205-214.

[57] P.T. Lee, R.G. Compton, Electrochemical detection of NADH, cysteine, or glutathione using a caffeic acid modified glassy carbon electrode, Electroanal. 25 (2013) 1613-1620.

[58] M.H. Pournaghi-Azar, F. Ahour, Palladized aluminum electrode covered by Prussian blue film as an effective transducer for electrocatalytic oxidation and hydrodynamic amperometry of N-acetyl-cysteine and glutathione, J. Electroanal. Chem. 622 (2008) 22-28. 